\title{
Relationship Between Line Bisection Test Time and Hemispatial Neglect Prognosis in Patients With Stroke: A Prospective Pilot Study
}

\author{
Shinyoung Kwon, $\mathrm{MD}^{1,2}$, Wookyung Park, $\mathrm{MD}^{1,2}$, MinYoung Kim, MD, PhD ${ }^{1,2}$, Jong Moon Kim, $\mathrm{MD}^{1,2}$
}

${ }^{1}$ Department of Rehabilitation Medicine, CHA Bundang Medical Center, CHA University College of Medicine, Seongnam; ${ }^{2}$ Rehabilitation and Regeneration Research Center, CHA University, Pocheon, Korea

\begin{abstract}
Objective To determine the relationship between line bisection test (LBT) performance time and prognosis of hemispatial neglect (HSN) in stroke patients.

Methods Data on stroke patients with HSN were prospectively collected. After patient recruitment and eligibility screening, the LBT, Motor-Free Visual Perception Test 3rd edition, and Korean version of Mini-Mental State Examination were performed at the time of admission and 4 weeks thereafter. The LBT performance time was also measured. All patients received conventional rehabilitation for 4 weeks. Based on the improvements in their LBT grades, the patients were divided into improved and non-improved groups. The evaluation results of the two groups were compared using Mann-Whitney U-tests and logistic regression was performed to predict the independence of each outcome.

Results In total, 26 stroke patients with HSN were included, with 13 patients in each group. Significant differences were observed in the baseline LBT performance times between the improved and non-improved groups $(\mathrm{p}<0.05)$. Logistic regression analysis revealed associations between HSN prognosis, and baseline LBT performance time (odds ratio $=0.95 ; 95 \%$ confidence interval, 0.90-1.00; $\mathrm{p}<0.05$ ) and baseline Motor-Free Visual Perception Test 3rd edition (odds ratio $=1.20 ; 95 \%$ confidence interval, 1.01-1.43; $\mathrm{p}<0.05$ ).

Conclusion A significant relationship was observed between the baseline LBT performance time and HSN prognosis.
\end{abstract}

Keywords Stroke, Perceptual disorders, Visual perception, Attention, Prognosis, Hemispatial neglect

\footnotetext{
Received August 2, 2019; Revised September 23, 2019; Accepted October 18, 2019; Published online August 5, 2020 Corresponding author: Jong Moon Kim

Department of Rehabilitation Medicine, CHA Bundang Medical Center, CHA University College of Medicine, 59 Yatap-ro, Bundang-gu, Seongnam 13496, Korea. Tel: +82-31-780-5456, Fax: +82-31-780-3449, E-mail: khan1013@chamc.co.kr

ORCID: Shinyoung Kwon (http://orcid.org/0000-0002-6888-8064); Wookyung Park (http://orcid.org/0000-0002-8939-0724); MinYoung Kim (http:// orcid.org/0000-0001-5481-2985); Jong Moon Kim (http://orcid.org/0000-0002-8684-8736).
}

(c) This is an open-access article distributed under the terms of the Creative Commons Attribution Non-Commercial License (http://creativecommons.org/ licenses/by-nc/4.0) which permits unrestricted noncommercial use, distribution, and reproduction in any medium, provided the original work is properly cited. Copyright $\odot 2020$ by Korean Academy of Rehabilitation Medicine 


\section{INTRODUCTION}

Hemispatial neglect (HSN) is characterized by reduced awareness of stimuli from the opposite side of the brain lesion [1]. HSN is closely related to damage to the temporoparietal junction and the posterior parietal cortex of the brain area which is associated with attention to the contralateral space. The lack of attention in the contralateral space can occur in the visual, auditory, proprioceptive, and olfactory areas, and is characterized by a lack of awareness of sensory input [2]. HSN is not uncommon in patients with right hemispheric lesions, occurring in $13 \%-81 \%$ of cases $[3,4]$. HSN patients experience difficulties in performing daily activities and may not be able to detect dangerous objects located on the affected side [5]. These issues can make post-stroke rehabilitation difficult and prevent functional recovery [6]. Therefore, HSN diagnosis and prognosis prediction are important for achiev- ing rehabilitation [7].

The evaluation of HSN in stroke patients is important and various tests have been developed for this purpose [8]. The most commonly used pencil-and-paper tests are the line bisection test (LBT) [9], clock drawing test [10], Albert test [11], and star cancellation test [12]. In particular, the LBT is a simple and less time-consuming test that is widely used in the clinical setting, with a relative test sensitivity of $76.4 \%$ for visuospatial neglect [13]. However, grade assessment by LBT in HSN includes only the deviation from the evaluation result and the performance time is not considered [14]. HSN after stroke is significantly improved in $43 \%$ of cases within 2 weeks and $63 \%$ of cases are nearly recovered within 3 months [15]. HSN is associated with various cognitive functions in the patients; therefore, many studies have assessed the relationships between neglect and various cognitive functions [16]. Previous studies demonstrated the relationship between neglect

Table 1. Demographics and clinical characteristics of patients

\begin{tabular}{|c|c|c|c|c|}
\hline Variable & $\begin{array}{c}\text { Total } \\
(\mathbf{n}=\mathbf{2 6})\end{array}$ & $\begin{array}{c}\text { Improved group }^{a)} \\
(n=13)\end{array}$ & $\begin{array}{c}\text { Non-improved group } \\
(n=13)\end{array}$ & p-value \\
\hline Age (yr) & $60.4 \pm 10.5$ & $57.6 \pm 11.2$ & $63.2 \pm 9.4$ & 0.15 \\
\hline Sex & & & & 0.67 \\
\hline Male & 18 & 8 & 10 & \\
\hline Female & 8 & 5 & 3 & \\
\hline Etiology & & & & 0.43 \\
\hline Ischemic stroke & 15 & 6 & 9 & \\
\hline Hemorrhagic stroke & 11 & 7 & 4 & \\
\hline Onset to baseline evaluation (day) & $41.8 \pm 87.0$ & $56.6 \pm 122.2$ & $27.0 \pm 18.6$ & 0.55 \\
\hline Side of brain lesion & & & & 0.44 \\
\hline Right & 19 & 10 & 9 & \\
\hline Left & 3 & 2 & 1 & \\
\hline Bilateral & 4 & 1 & 3 & \\
\hline Location of brain lesion & & & & 0.37 \\
\hline Frontal & 13 & 6 & 7 & \\
\hline Parietal & 9 & 3 & 6 & \\
\hline Temporal & 5 & 2 & 3 & \\
\hline Others ${ }^{\mathrm{b})}$ & 13 & 8 & 5 & \\
\hline Baseline K-MBI score & $50.4 \pm 22.8$ & $50.0 \pm 23.8$ & $50.8 \pm 22.7$ & 1.00 \\
\hline Received rTMS & 5 & 2 & 3 & \\
\hline
\end{tabular}

Values are presented as mean \pm standard deviation.

K-MBI, Korean version of Modified Barthel Index; rTMS, repetitive transcranial magnetic stimulation.

${ }^{a)}$ Improved group is a group with line bisection test score change, and non-improved group is a group with no score change.

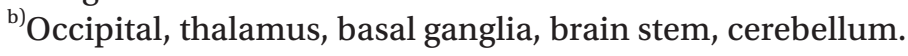


and cognitive impairment using the Mini-Mental State Examination (MMSE), while the severity of neglect was assessed using visual perception tests such as the MotorFree Visual Perception Test (MVPT) [17,18]. HSN patients reportedly have greater deterioration in attention $[19,20]$, which is related to the duration of the cognitive function test and test performance time $[21,22]$. The present study investigated whether LBT performance time could predict the HSN prognosis and to confirm the relationship between MMSE and MVPT-3 (MVPT 3rd edition).

\section{MATERIALS AND METHODS}

\section{Subjects}

We prospectively enrolled all patients with a first acute stroke admitted to the Department of Rehabilitation Medicine of CHA Bundang Medical Center between December 2017 and March 2019. Patients were included: (1) who experienced a stroke as confirmed by a neurologist using computed tomography or magnetic resonance imaging, (2) were aged $\geq 20$ years, (3) were able to perform at least a one-step obey command, (4) had motor power of at least one upper limb higher than a fair grade on the manual muscle test on admission (as fair and below fair motor grades could interfere with the evaluation test), and (5) who were diagnosed with HSN using the LBT. Subjects (1) with an underlying neurological condition other than stroke such as brain tumor, traumatic brain injury, and hydrocephalus; (2) with a history of diseases that can affect visual function such as ophthalmologic disease; and (3) in whom evaluation was impossible because they could not sit independently were excluded. The study was conducted according to the principles of the Helsinki Declaration and was approved by the Institutional Review Board of CHA Bundang Medical Center
(No. CHAMC2017-11-037). All patients were provided written and oral information about the study and all patients provided their consent before study participation.

All patients received neglect treatment such as visual scanning, prism adaptation, biofeedback training, and limb activation depending on their function. Five patients received repetitive transcranial magnetic stimulation treatment for cognitive improvement (Table 1).

\section{Evaluation methods}

Based on the improvement in LBT grade (from that at baseline to that at 4 weeks thereafter), the patients were divided into improved and non-improved groups. The patients were allocated to the improved group when the LBT grade was lower than that at baseline (that measured at admission); if the baseline LBT grade was not lower than that at baseline, the patient was allocated to the non-improved group.

All the evaluations were performed by an experienced occupational therapist and reviewed by a rehabilitation specialist. The following evaluation tests were performed 4 weeks after the baseline evaluation.

\section{Primary outcome measure \\ $L B T$}

The LBT is a tool proposed by Schenkenberg et al. [9] for evaluating unilateral neglect. On the test paper are 20 lines in a random arrangement, with 8 lines in the center, 6 on the left, and 6 on the right. The lines are 100, 120, 140, 160, 180, and $200 \mathrm{~mm}$ in length (Fig. 1). In this study, the A4-size test paper was placed on the patient's center line with the patient in a sitting position. The examiner first showed the center point of the two top and bottom lines and instructed the patient to mark the center points of the remaining 18 lines. The top and bottom demon-

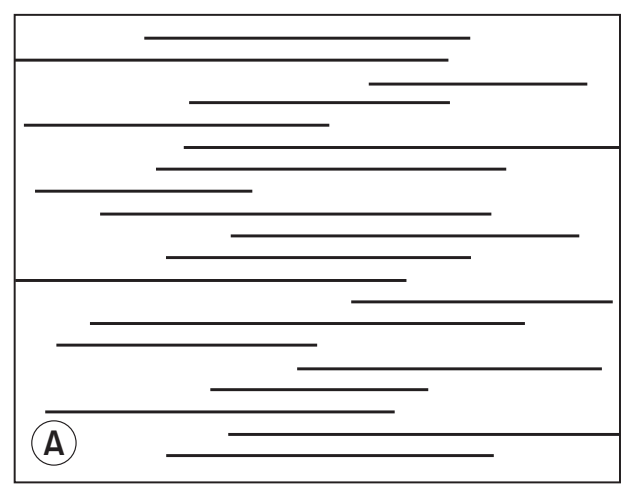

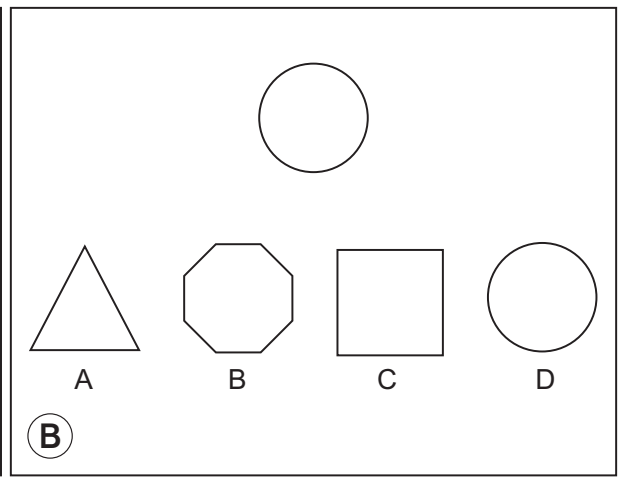

Fig. 1. Examples of evaluation tool. (A) Line bisection test consists of 20 horizontal lines and (B) Motor-Free Visual Perception Test is a multiple-choice test that can evaluate spatial relationship, visual discrimination, figureground discrimination, visual closure, and visual memory. 
stration lines were excluded when measuring scores. The test ended when the patient believed that he/she had completed the task and placed the pen on the desk. In this study, the test time was measured using a stopwatch and there was no time limit.

The severity of HSN was classified as follows in this study. We calculated the mean of the 6 left and 6 center straight lines and calculated the standard deviation for each patient using the following formula:

$\frac{\text { Patient mean-Normal mean }}{\text { Normal standard deviation }}=$ Patient standard deviation

The mean value of normal subjects was -0.59 , with a standard deviation of 4.35. A standard deviation absolute value $>1$ indicated mild neglect (grade 1 ), $>2$ indicated moderate neglect (grade 2), and $>3$ indicated severe neglect (grade 3). Patients with a standard deviation absolute value $<1$ were classified as normal [14].

\section{Secondary outcome measure MVPT-3}

The MVPT-3 is a tool for evaluating visual perception ability without using motor function (Fig. 1). The MVPT was first developed in the 1970s; the present study used the MVPT-3 revision from 2003. The preliminary reliability and validity have been reported and its validity has been reported using Rasch analysis [23,24]. The MVPT-3 assesses the overall perception of spatial relationships, visual discrimination, figure-ground discrimination, visual closure, and visual memory, and is divided into the following eight sub-areas: visual discrimination, form constancy, visual short-term memory 1 , visual closure 1 , spatial orientation, figure ground, visual closure 2 , and visual short-term memory [23]. The test comprises 65 items; for patients aged $\geq 11$ years, only items 14-65 are evaluated. In this study, the raw score was used in the evaluation without using the conversion score according to age.

\section{Korean version of the MMSE}

Of several MMSE translations used in Korea, this study used the K-MMSE. The K-MMSE comprises five points of orientation for time (5 points), orientation for place (5 points), registration ( 3 points), attention and calculation (5 points), recall ( 3 points), language (8 points), and visual construction ( 1 point). The total score is 30 points [25]. In this study, the differences between the scores at baseline and after 4 weeks were verified using the total score.

\section{Statistical analyses}

Differences in the baseline characteristic variables between the patient groups were analyzed by age, onset to baseline evaluation, Korean version of the Modified Barthel Index score using Mann-Whitney U-tests, sex, and etiology using Fisher exact tests; the side and location of the brain lesions were analyzed using linear by linear association. Mann-Whitney U-tests were conducted to compare the baseline and changes in LBT time, MVPT-3, and K-MMSE between the two groups.

Logistic regression analysis was conducted to identify the prognostic factors of HSN from baseline. The included factors were age, sex, onset to baseline evaluation day, side of the brain lesion, location of the brain lesion (frontal, parietal, and temporal lobe), baseline LBT time, baseline MVPT-3 score, and baseline MMSE score.

Statistical analyses were performed using standard statistical software (SPSS version 21.0 for Windows; IBM, Armonk, NY, USA). The significance level was set at below 0.05 .

\section{RESULTS}

\section{Baseline subject characteristics}

A total of 39 patients were recruited. Six were lost to follow-up; of the remaining patients, 7 who received grade 0 in the baseline LBT were excluded from the statistical analysis (Fig. 2). Finally, 26 stroke patients (18 men and 8 women; mean age, $60.4 \pm 10.5$ years) with HSN were included (15 with ischemic stroke and 11 with

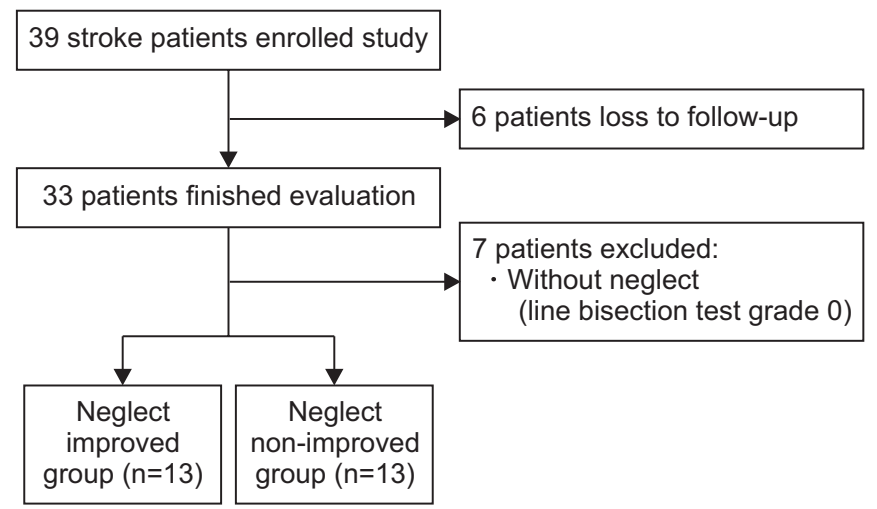

Fig. 2. Flowchart of the study. 
hemorrhagic stroke). According to the change in the LBT grade, the patients were divided into the improved $(n=13)$ and non-improved $(n=13)$ groups. The times from onset to baseline evaluation were $56.6 \pm 122.2$ days in the improved group and 27.0 \pm 18.6 days in the non-improved group. All patients showed the times from onset to baseline evaluation within 90 days, except for one patient in the improved group ( 460 days). Table 1 summarizes the demographic and clinical characteristics of the patients. No statistically significant differences were observed between the two groups.

Comparisons between improved and non-improved HSN groups

Comparisons of baseline LBT performance times and changes in LBT performance time after 4 weeks

A significant difference was observed in the baseline LBT performance time of both groups $(\mathrm{p}<0.05)$. However, the changes in the LBT performance time after 4 weeks were not significantly different ( $p>0.05$ ) (Table 2, Fig. 3).

Comparison of baseline MVPT-3 score and changes in MVPT-3 score after 4 weeks

No significant differences were observed in the baseline MVPT-3 and changes in MVPT-3 scores of the two groups (p>0.05) (Table 2, Fig. 3).

Comparisons of the baseline K-MMSE total scores and changes in the scores at 4 weeks

No significant differences were observed in the baseline K-MMSE and changes in K-MMSE scores of the two groups ( $\mathrm{p}>0.05$ ) (Table 2, Fig. 3).

\section{Logistic regression analysis}

Logistic regression analysis performed for each variable in all the subjects showed that sex, baseline LBT time, and baseline MVPT-3 score were significantly associated with HSN (Table 3).

Table 2. Comparison of LBT, K-MMSE and MVPT-3 results between two groups

\begin{tabular}{|c|c|c|c|}
\hline Variable & Improved group (n=13) & Non-improved group $(n=13)$ & p-value \\
\hline \multicolumn{4}{|l|}{ LBT grade } \\
\hline Baseline evaluation & & & 0.63 \\
\hline Grade 0 (normal) & 0 & 0 & \\
\hline Grade 1 (mild) & 9 & 10 & \\
\hline Grade 2 (moderate) & 1 & 1 & \\
\hline Grade 3 (severe) & 3 & 2 & \\
\hline After 4 weeks evaluation & & & $0.00^{* *}$ \\
\hline Grade 0 (normal) & 10 & 0 & \\
\hline Grade 1 (mild) & 2 & 8 & \\
\hline Grade 2 (moderate) & 0 & 2 & \\
\hline Grade 3 (severe) & 0 & 3 & \\
\hline \multicolumn{4}{|l|}{ LBT time (sec) } \\
\hline Baseline LBT time & $61.45 \pm 25.12$ & $101.15 \pm 58.57$ & $0.03^{*}$ \\
\hline$\Delta$ LBT time & $10.85 \pm 16.94$ & $30.49 \pm 65.30$ & 0.58 \\
\hline \multicolumn{4}{|l|}{ MVPT-3 } \\
\hline Baseline MVPT-3 score & $41.00 \pm 11.14$ & $28.46 \pm 17.60$ & 0.20 \\
\hline$\Delta \mathrm{MVPT}-3$ score & $6.69 \pm 6.01$ & $11.92 \pm 12.95$ & 0.45 \\
\hline \multicolumn{4}{|l|}{ K-MMSE } \\
\hline Baseline K-MMSE score & $23.00 \pm 4.69$ & $19.69 \pm 5.14$ & 0.15 \\
\hline$\Delta \mathrm{K}$-MMSE score & $3.77 \pm 2.98$ & $5.62 \pm 2.53$ & 0.07 \\
\hline
\end{tabular}

Values are presented as mean \pm standard deviation.

LBT, line bisection test; MVPT-3, Motor-Free Visual Perception Test 3rd edition; K-MMSE, Korean version of MiniMental State Examination.

${ }^{*} \mathrm{p}<0.05,{ }^{* *} \mathrm{p}<0.01$. 

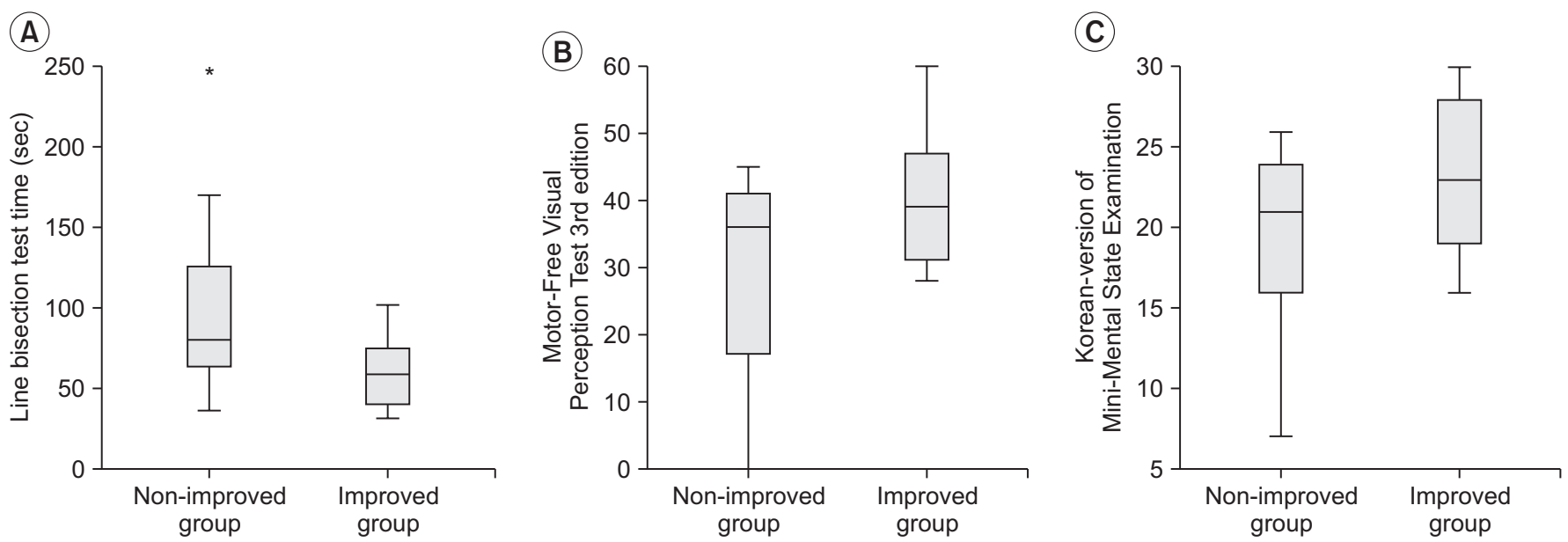

Fig. 3. Comparison of results between two groups. (A) Line bisection test time, (B) Motor-Free Visual Perception Test 3rd edition, and (C) Korean version of Mini-Mental State Examination. * $\mathrm{p}<0.05$.

Table 3. Logistic regression analysis for prognosis of hemispatial neglect

\begin{tabular}{|lrll|}
\hline \multicolumn{1}{|c}{ Variables } & OR & $\mathbf{9 5 \%}$ CI & p-value \\
\hline Age (yr) & 0.95 & $0.82-1.10$ & 0.48 \\
\hline Sex, male & 48.68 & $1.20-1,967.42$ & $0.04^{*}$ \\
\hline Onset to baseline evaluation (day) & 1.11 & $1.00-1.24$ & 0.06 \\
\hline Side of brain lesion, right & 1.95 & $0.01-414.86$ & 0.81 \\
\hline Location of brain lesion & & & \\
\hline Frontal & 40.97 & $0.22-7,704.45$ & 0.16 \\
\hline Parietal & 0.00 & $0.00-1.71$ & 0.07 \\
\hline Temporal & 212.76 & $0.11-400,574.19$ & 0.16 \\
\hline Baseline LBT time (sec) & 0.95 & $0.90-1.00$ & $0.04^{*}$ \\
\hline Baseline MVPT-3 score & 1.20 & $1.01-1.43$ & $0.04^{*}$ \\
\hline Baseline K-MMSE score & 1.36 & $0.74-2.48$ & 0.32 \\
\hline
\end{tabular}

OR, odd ratio; CI, confidence interval; LBT, line bisection test; MVPT-3, Motor-Free Visual Perception Test 3rd edition; K-MMSE, Korean version of Mini-Mental State Examination.

${ }^{*} \mathrm{p}<0.05$.

\section{DISCUSSION}

This study aimed to determine whether the LBT performance time, which corresponds to sustained attention, is related to HSN prognosis. The results demonstrated a shorter baseline LBT performance time, higher proportion of male sex, and higher baseline MVPT-3 score in the improved group. Therefore, HSN was more likely to reduce when the test performance time was short because the LBT was conducted more intensively.

Many theories have been proposed to explain the characteristic behaviors of HSN, the most widely accepted of which is the attention-based theory. HSN is believed to be caused by an abnormality in the brain network that results in a lack of attention control $[26,27]$. Therefore, the maintenance of attention is accompanied by lower arousal and speed processing; this not only reduces task performance but also decreases the response to the stimulus at rest $[27,28]$. The present study measured the test performance time to confirm patient attention. The LBT is a simple and clinically useful test for HSN for which various detailed test methods and analyses have been developed [29]. However, in general, there is little consideration of the time concept when analyzing the LBT. Most studies use the average of several straight lines; however, the number, length, and position of the lines on the sheet 
of paper differs lightly. Some studies have used the concept of time to interpret results, in which performance time $>90$ seconds is indicative of severe-grade neglect [30]. The introduction of this time concept may be useful to determine the HSN severity.

Various HSN assessment tools have been developed in which various tasks are performed and evaluated. One assessment of the method of interpretation contains the concept of time (to the extent that it is performed with a time limit); however, most methods do not use the concept of time [31]. In a study using a cancellation test conducted on a touch screen, the HSN group was slower and less efficient than the control group [32]. Among patients with the same degree of severity in the previous assessment, patient who with severe loss of concentration may have a delayed performance time. Therefore, incorporation of the time concept in popularized tests such as the LBT and star calculation tests may allow a more comprehensive assessment. HSN can be accompanied by cognitive impairments in attention, memory, visual perception, and visual-motor organization [33]. Previous studies reported that patients with poor outcomes of HSN had significantly lower visual perception and memory function [34] and chronic HSN was related to sustained inattention [35]. In our study, shorter baseline LBT performance time and higher baseline MVPT-3 results had a more positive effect on patient outcomes. Therefore, cognitive impairment such as attention and visual perception affects HSN recovery in patients.

HSN patients may miss or perceive distorted information in the visual hemifield on the opposite side of the lesion during evaluation. This phenomenon makes most perceptual tests difficult for HSN patients. Some studies have developed a version of the MVPT with vertically aligned answers to eliminate these effects [36]. Rather, these tests remove the HSN variable that involves the disadvantage of not being usable for evaluating activities of daily living such as driving [37]. Our results showed no significant differences in the baseline MVPT-3 scores of both groups. However, the logistic regression results indicated that the higher the baseline MVPT-3 score, the better the prognosis. Thus, patients with the same HSN severity who had worse prognoses are expected to have lower baseline MVPT-3 scores.

The MMSE was originally developed to diagnose dementia and has a variety of cognitive subdomains $[38,39]$.
In previous studies, patients with neglect mostly showed decreased time orientation, attention, and recall on the MMSE $[17,40]$. The present study observed no statistically significant differences in MMSE total score. In future studies, it would be helpful to target more patients and use cognitive assessments with reliable subdomain variables.

This study has several limitations. First, the stroke patients who participated in this study received conventional rehabilitation for 4 weeks between the baseline and the follow-up evaluations. Therefore, there was the possibility of bias introduced by the treatment intervention. However, cognitive and neglect treatments were administered similarly as per the patient's functional level; therefore, the results are not expected to have a significant effect. Second, the number of patients was small and long-term prognosis evaluation is needed. Third, further study is needed to assess the relationship between elevated LBT grade and prognosis. In this study, the LBT grades were analyzed based on increase or maintenance and grade decrease. The grade change from 2 to 1 and from 3 to 1 can differ; however, a small number of samples made it difficult to finely classify the groups. Future studies should enroll more patients and perform comparisons among patients with different degrees of HSN severity.

In conclusion, the LBT is one of the most commonly used testing tools in HSN patients. This study observed a significant association between LBT performance time and HSN prognosis. In addition, the baseline MVPT3 score can be considered in predicting prognosis. The measurement of LBT performance time is simple and can be used to predict the prognosis of HSN patients.

\section{CONFLICT OF INTEREST}

No potential conflict of interest relevant to this article was reported.

\section{ACKNOWLEDGMENTS}

This work was supported by a grant from the Korea Health Technology R\&D Project through the Korea Health Industry Development Institute (KHIDI), funded by the Ministry of Health \& Welfare, Republic of Korea (No. HI16C1559). 


\section{AUTHOR CONTRIBUTION}

Conceptualization: Kim JM, Kwon S. Methodology: Kim JM, Kim M, Kwon S. Formal analysis: Kim JM, Kwon S. Project administration: Kim JM. Visualization: Kwon S, Park W. Writing - original draft: Kwon S, Park W. Writing - review and editing: Kim JM, Kim M. Approval of final manuscript: all authors.

\section{REFERENCES}

1. Heilman KM, Bowers D, Coslett HB, Whelan H, Watson RT. Directional hypokinesia: prolonged reaction times for leftward movements in patients with right hemisphere lesions and neglect. Neurology 1985;35: 855-9.

2. Vallar G. Spatial hemineglect in humans. Trends Cogn Sci 1998;2:87-97.

3. Bisiach E, Perani D, Vallar G, Berti A. Unilateral neglect: personal and extra-personal. Neuropsychologia 1986;24:759-67.

4. Stone SP, Wilson B, Wroot A, Halligan PW, Lange LS, Marshall JC, et al. The assessment of visuo-spatial neglect after acute stroke. J Neurol Neurosurg Psychiatry 1991;54:345-50.

5. Denes G, Semenza C, Stoppa E, Lis A. Unilateral spatial neglect and recovery from hemiplegia: a followup study. Brain 1982;105:543-52.

6. Jehkonen M, Ahonen JP, Dastidar P, Koivisto AM, Laippala P, Vilkki J, et al. Visual neglect as a predictor of functional outcome one year after stroke. Acta Neurol Scand 2000;101:195-201.

7. Appelros P, Karlsson GM, Seiger A, Nydevik I. Prognosis for patients with neglect and anosognosia with special reference to cognitive impairment. J Rehabil Med 2003;35:254-8.

8. Azouvi P, Samuel C, Louis-Dreyfus A, Bernati T, Bartolomeo P, Beis JM, et al. Sensitivity of clinical and behavioural tests of spatial neglect after right hemisphere stroke. J Neurol Neurosurg Psychiatry 2002;73: 160-6.

9. Schenkenberg T, Bradford DC, Ajax ET. Line bisection and unilateral visual neglect in patients with neurologic impairment. Neurology 1980;30:509-17.

10. Ishiai S, Sugishita M, Ichikawa T, Gono S, Watabiki S. Clock-drawing test and unilateral spatial neglect.
Neurology 1993;43:106-10.

11. Albert ML. A simple test of visual neglect. Neurology 1973;23:658-64.

12. Friedman PJ. The star cancellation test in acute stroke. Clinical Rehabil 1992;6:23-30.

13. Bailey MJ, Riddoch MJ, Crome P. Evaluation of a test battery for hemineglect in elderly stroke patients for use by therapists in clinical practice. NeuroRehabilitation 2000;14:139-50.

14. Van Deusen J. Normative data for ninety-three elderly persons on the Schenkenberg line bisection test. Phys Occup Ther Geriatr 1985;3:49-54.

15. Farne A, Buxbaum LJ, Ferraro M, Frassinetti F, Whyte J, Veramonti T, et al. Patterns of spontaneous recovery of neglect and associated disorders in acute right braindamaged patients. J Neurol Neurosurg Psychiatry 2004;75:1401-10.

16. Halligan PW, Fink GR, Marshall JC, Vallar G. Spatial cognition: evidence from visual neglect. Trends Cogn Sci 2003;7:125-33.

17. Lee BH, Kim EJ, Ku BD, Choi KM, Seo SW, Kim GM, et al. Cognitive impairments in patients with hemispatial neglect from acute right hemisphere stroke. Cogn Behav Neurol 2008;21:73-6.

18. Yi YG, Chun MH, Do KH, Sung EJ, Kwon YG, Kim DY. The effect of transcranial direct current stimulation on neglect syndrome in stroke patients. Ann Rehabil Med 2016;40:223-9.

19. Weinberg J, Diller L, Gerstman L, Schulman P. Digit span in right and left hemiplegics. J Clin Psychol 1972; 28:361.

20. Robertson I. Anomalies in the laterality of omissions in unilateral left visual neglect: implications for an attentional theory of neglect. Neuropsychologia 1989; 27:157-65.

21. Stuss DT, Stethem LL, Hugenholtz H, Picton T, Pivik J, Richard MT. Reaction time after head injury: fatigue, divided and focused attention, and consistency of performance. J Neurol Neurosurg Psychiatry 1989;52: 742-8.

22. Paus T, Zatorre RJ, Hofle N, Caramanos Z, Gotman J, Petrides M, et al. Time-related changes in neural systems underlying attention and arousal during the performance of an auditory vigilance task. J Cogn Neurosci 1997;9:392-408.

23. Brown GT, Rodger S, Davis A. Motor-free visual per- 
ception test-revised: an overview and critique. British Journal of Occupational Therapy 2003;66:159-67.

24 . Brown T. An examination of the construct validity of the Motor-Free Visual Perceptual Test-Third Edition (MVPT-3) using Rasch analysis with adult participants. OTJR 2001;31:73-80.

25. Kang Y, Na DL, Hahn S. A validity study on the Korean mini-mental state examination (K-MMSE) in dementia patients. J Korean Neurol Assoc 1997;15:300-8.

26. Mesulam MM. Spatial attention and neglect: parietal, frontal and cingulate contributions to the mental representation and attentional targeting of salient extrapersonal events. Philos Trans R Soc Lond B Biol Sci 1999;354:1325-46.

27. Corbetta M, Shulman GL. Spatial neglect and attention networks. Annu Rev Neurosci 2011;34:569-99.

28. Whyte J. Attention and arousal: basic science aspects. Arch Phys Med Rehabil 1992;73:940-9.

29. Jang WH. Status of occupational therapists on unilateral neglect test tools usage and symptom classification. J Korean Phys Ther 2017;29:271-5.

30. Cha YJ, Kim K. The comparison of plantar pressure distribution regarding the extent of hemineglect in adult hemiplegia. J Korean Soc Phys Med 2010;5:4351.

31. Yoo WK. Subtypes of spatial neglect and assessment. Brain Neurorehabil 2009;2:46-50.

32. Rabuffetti M, Farina E, Alberoni M, Pellegatta D, Appollonio I, Affanni P, et al. Spatio-temporal features of visual exploration in unilaterally brain-damaged subjects with or without neglect: results from a touch- screen test. PLoS One 2012;7:e31511.

33. Paolucci S, Antonucci G, Grasso MG, Pizzamiglio L. The role of unilateral spatial neglect in rehabilitation of right brain-damaged ischemic stroke patients: a matched comparison. Arch Phys Med Rehabil 2001; 82:743-9.

34. Jeong EH, Kim BR, Lee J. Relationship between comorbid cognitive impairment and functional outcomes in stroke patients with spatial neglect. Brain Neurorehabil 2016;9:37-47.

35. Hjaltason H, Tegner R, Tham K, Levander M, Ericson K. Sustained attention and awareness of disability in chronic neglect. Neuropsychologia 1996;34:1229-33.

36. Mercier L, Hebert R, Gauthier L. Motor Free Visual Perceptual Test: impact of vertical answer cards position on performance of adults with hemispatial visual neglect. OTJR 1995;15:223-36.

37. Mazer BL, Korner-Bitensky NA, Sofer S. Predicting ability to drive after stroke. Arch Phys Med Rehabil 1998;79:743-50.

38. Folstein MF, Folstein SE, McHugh PR. "Mini-mental state". A practical method for grading the cognitive state of patients for the clinician. J Psychiatr Res 1975; 12:189-98.

39. Tombaugh TN, McIntyre NJ. The mini-mental state examination: a comprehensive review. J Am Geriatr Soc 1992;40:922-35.

40. Wade DT, Wood VA, Hewer RL. Recovery of cognitive function soon after stroke: a study of visual neglect, attention span and verbal recall. J Neurol Neurosurg Psychiatry 1988;51:10-3. 